\title{
Infection, reinfection, and vaccination under suboptimal immune protection: epidemiological perspectives
}

\author{
a Instituto Gulbenkian de Ciência, Apartado 14, 2781-901 Oeiras, Portugal \\ ${ }^{\mathrm{b}}$ Ecology and Epidemiology Group, Department of Biological Sciences, University of Warwick, Coventry, CV4 7 AL, UK
}

\section{Abstract}

The SIR (susceptible-infectious-resistant) and SIS (susceptible-infectious-susceptible) frameworks for infectious disease have been extensively studied and successfully applied. They implicitly assume the upper and lower limits of the range of possibilities for host immune response. However, the majority of infections do not fall into either of these extreme categories. We combine two general avenues that straddle this range: temporary immune protection (immunity wanes over time since infection), and partial immune protection (immunity is not fully protective but reduces the risk of reinfection). We present a systematic analysis of the prnamics and equilibrium properties of these models in comparison to SIR and SIS, and analyse the outcome of vaccination programmes. We describe how the waning of immunity shortens inter-epidemic periods, and poses major difficulties to disease eradication. We identify a "reinfection threshold" in transmission when partial immunity is included. Below the reinfection threshold primary infection dominates, levels of infection are low, and vaccination is highly effective (approximately an SIR model). Above the reinfection threshold reinfection dominates, levels of infection are high, and vaccination fails to protect (approximately an SIS situation). This association between high prevalence of infection and vaccine failure emphasizes the problems of controlling recurrent infections in high-burden regions. However, vaccines that induce a better protection than natural infection have the potential to increase the reinfection threshold, and therefore constitute interventions with a surprisingly high capacity to reduce infection where reduction is most needed.

\section{Introduction} 407-970.
Mathematical epidemiological models for the dynamics of microparasite infections that induce lifelong immunity have been extensively developed (Kermack and McKendrick, 1927; Anderson and May, 1991; Grenfell et al., 2001) and used as predictive tools to assist in the design of control programmes (Osborne et al., 2000). This class of infections is viral and usually occurs during childhood (if there is no vaccination occurring), which is indicative of high transmissibility.

Such effective immunity is observed in infections such as measles, mumps and rubella (MMR) but this is unusual. More common is the occurrence of several reinfections throughout life. In similar vein to char-

*Corresponding author. Tel.: +351-214-464-626; fax: +351-214-

E-mail address: ggomes@igc.gulbenkian.pt (M.G.M. Gomes). acterization of a vaccine response (McLean and Blower, 1993), susceptibility to reinfection following a primary infection can be attributed to a combination of two factors: immune protection may wane over time (temporary immunity), or immunity may not be fully protective (partial immunity). Both effects are likely due to a combination of host insufficiency in acquiring and maintaining specific immunity (e.g. tuberculosis-Vynnycky and Fine, 1997; pertussis-Hethcote, 1999; van Boven et al., 2000) and of pathogen ability to generate antigenic diversity thereby avoiding immune recognition (e.g. influenza-Hay et al., 2001; Earn et al., 2002; respiratory syncytial virus - Cane, 2001).

The specific within-host mechanisms allowing recurrent infections are not explicit in this paper. Rather, we construct a series of simple models to investigate the epidemiological consequences of varying the duration and degree of immune protection. The models are 
1 systematically analysed and reveal two main outcomes: the duration of immunity has a crucial impact on 3 potential inter-epidemic periods; the degree of immune protection determines a reinfection threshold in trans-

5 mission responsible for a steep increase in the prevalence of infection. The models are then extended to analyse

7 the effects of temporary and partial immunity on the global impact of vaccination programmes.

9 Mathematical models have long been associated with the planning of vaccination programmes. The main

11 contributions have been the prediction of the vaccination coverage necessary to eradicate infections (Nokes

13 and Anderson, 1992, 1993), and the illustration of the post-vaccination dynamics if coverage does not meet the eradication threshold (McLean, 1995a). McLean and Blower (1993) have also investigated the consequences of vaccine failures, but under the assumption that immunity induced by natural infection was fully

19 protective. Here this assumption is relaxed to accommodate the recurrence of infections. In the case of

21 temporary protection, we reach the same basic conclusion that the waning of immunity is a major obstacle to

23 disease eradication even if individuals are protected until very late in their lifetime. In the case of partial due to reinfection threshold. Below the reinfection

27 threshold, levels of infection are low and vaccination impact is high. The transmission dynamics are essentially described by the susceptible-infected-recovered $(S I R)$ framework. Populations where transmissibility is 31 above the reinfection threshold are of greater concern. They sustain very high endemicities and vaccination programmes are unable to increase natural immunity further. The transmission dynamics are essentially

35 susceptible-infected-susceptible $(S I S)$. The transition between the two regimes happens over a short range in 37 transmission coefficient, indicating high sensitivity to changes.

Throughout this paper we assume that birth and death rates are equal ensuring a constant host popula- assumed to mix homogeneously and transmission is according to the mass-action principle. Hosts are divided into three proportions: susceptible $(S)$, infectious $(I)$, and recovered $(R)$. Under the assumption that recovered individuals acquired some immunity that is totally protective and lifelong, we obtain the so-called SIR model formalized by the system of differential equations

$$
\begin{aligned}
& \frac{\mathrm{d} S}{\mathrm{~d} T}=\mu-\beta I S-\mu S, \\
& \frac{\mathrm{d} I}{\mathrm{~d} T}=\beta I S-(\tau+\mu) I, \\
& \frac{\mathrm{d} R}{\mathrm{~d} T}=\tau I-\mu R .
\end{aligned}
$$

As in Table 1, the parameter $\mu$ is the death rate (and equally, the birth rate), $\tau$ is the rate of recovery from infection, and $\beta$ is a transmission coefficient which combines a variety of epidemiological, environmental, and social factors that affect transmission. Susceptible individuals acquire infection at per capita rate $\beta I$. If time, $T$, is measured in years, hosts are born with a life expectancy of

$L=\frac{1}{\mu}$ years

and we assume throughout that $L=70$ years. The average duration of infection is

$D=\frac{1}{\tau+\mu}$ years

and we assume throughout that $D$ is approximately 1 month. The last equation of model (1) can be omitted by recalling that $S+I+R=1$.

\subsection{Non-dimensional SIR model}

The SIR model can be further simplified, resulting in a reduction in the number of parameters. Measuring time in units of duration of infection, $t=T / D$, we get the nondimensional system (see Table 2)

$\frac{\mathrm{d} S}{\mathrm{~d} t}=e-R_{0} I S-e S$,

$\frac{\mathrm{d} I}{\mathrm{~d} t}=R_{0} I S-I$.

The first parameter,

$e=\frac{D}{L}$

Table 1

Model parameters when time is measured in years

\begin{tabular}{lll}
\hline Symbol & Definition & Value \\
\hline$\mu$ & Death rate and, equally, birth rate & $1 / 70$ year $^{-1}$ \\
$\tau$ & Rate of recovery from infection & 12 year $^{-1}$ \\
$\beta$ & Transmission coefficient & Variable \\
\hline
\end{tabular}
result of infection experience. The population is 
1 Table 2

3
Model parameters when time is measured in units of average duration of infection, $D=1 /(\tau+\mu)$

(e)

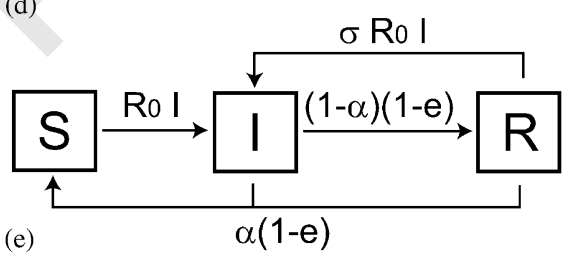

Fig. 1. Flow diagram for all the models analysed in this paper. Time is in units of average duration of infection and the parameters are described in Table 1. For simplicity, births and deaths are not represented.

for $i=1,2$. A straightforward calculation gives the following results:

(1) Eigenvalues of $J_{1}$ : $-e$ and $R_{0}-1$.

(2) Eigenvalues of $J_{2}: \frac{-e R_{0} \pm \sqrt{e^{2} R_{0}^{2}-4 e\left(R_{0}-1\right)}}{2}$.

The eigenvalues of $J_{1}$ are always real, and from their signs we infer that the steady state $\left(S_{1}, I_{1}\right)$ is stable for $R_{0}<1$, and unstable for $R_{0}>1$. The eigenvalues of $J_{2}$ have negative real part for the whole range of validity of the steady state $\left(S_{2}, I_{2}\right)$, implying that this steady state is always stable. The eigenvalues are real for $R_{0}$ just above 1 , and become complex for $R_{0}$ between $(2 / e)(1-$ $\sqrt{1-e})$ and $(2 / e)(1+\sqrt{1-e})$ implying convergence in the form of damped oscillations in this range (for $e=0.0012$, this is $1.0003<R_{0}<3332$ ). The vector field is illustrated in Fig. 2(a) for the particular case $R_{0}=3$. Superimposed is a simulation starting near the diseasefree equilibrium illustrating how the disease invades and converges to the endemic equilibrium. Fig. 2(b) shows the time-series plot corresponding to the same simulation. Convergence is in the form of damped oscillations: a series of epidemics of sequentially reducing amplitude occur until the system approaches the endemic equilibrium. A number of mechanisms have been identified as capable of sustaining the oscillations (Liu et al., 1987), enhancing the importance of quantifying the interepidemic periods.

\subsection{Non-dimensional SIS model}

103

Variations of the SIR model have been developed to incorporate features of particular diseases (Anderson and May, 1991). Here, we consider diseases that fail to elicit protective immunity, allowing recurrent infections. In this extreme case, recovered hosts return to the susceptibility class and the recovered class remains empty so that $S+I=1$. The dynamics of this system are driven by a one-dimensional system: the SIS model,
105

107

109

111 
$51 \frac{\mathrm{d} I}{\mathrm{~d} t}=R_{0} I(1-I)-I$.

53 Also here, there is a disease-free equilibrium $(S=1, I=$ 0 ) for all values of $R_{0}$. Stability analysis shows that this
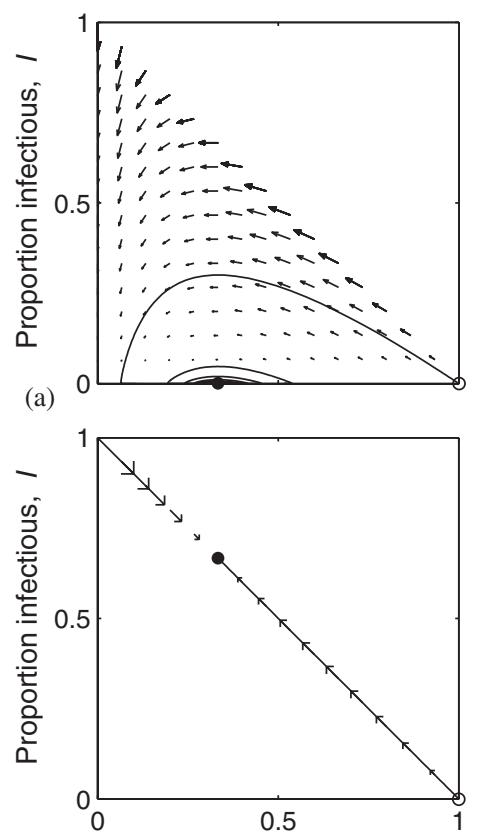

(c) Proportion susceptible, $S$
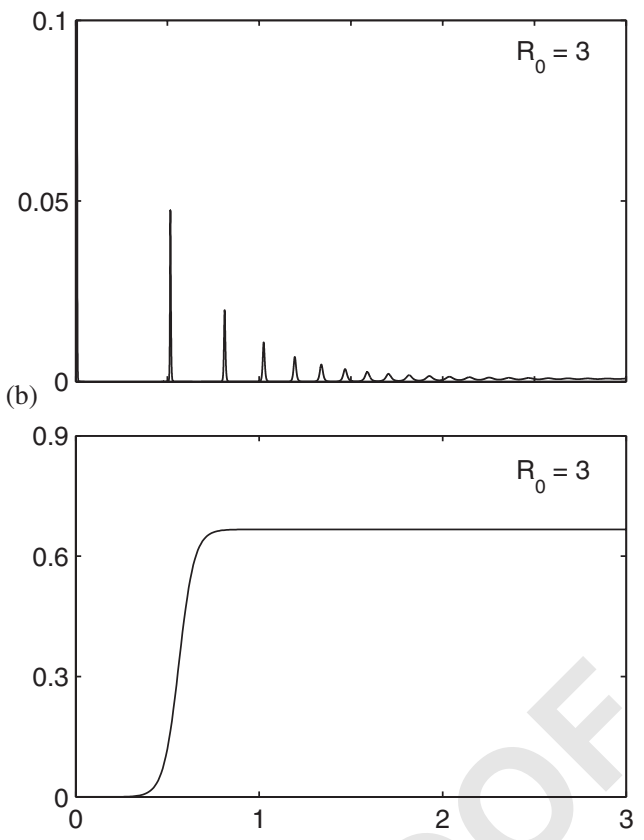

(d) equilibria bifurcates. At the endemic steady state we have $S=1 / R_{0}$ and $I=1-1 / R_{0}$. In Fig. 2(c) and (d), we fix $R_{0}=3$ to represent the vector field associated with the SIS model and a simulation to illustrate convergence to the steady state. The bifurcation diagrams corresponding to both the $S I R$ and $S I S$ models are shown as the full lines in Fig. 3, setting the lower and upper bounds to the intermediate models.

\section{Intermediate models}

\subsection{Temporary immune protection}

Pertussis is a highly contagious infection of the respiratory tract where recurrence has been attributed to the waning of immunity (Hethcote, 1999; van Boven et al., 2000). Control by vaccination is less successful than for other childhood diseases, and recent trends are for overall increase, and occasionally strong epidemic outbreaks.

The temporary immunity model is represented diagrammatically in Fig. 1(c). Our assumption is that, upon infection, individuals develop an immune response that is lost at a certain rate. The parameter $\alpha$ is introduced to control the rate of loss of immunity. The model equations are 

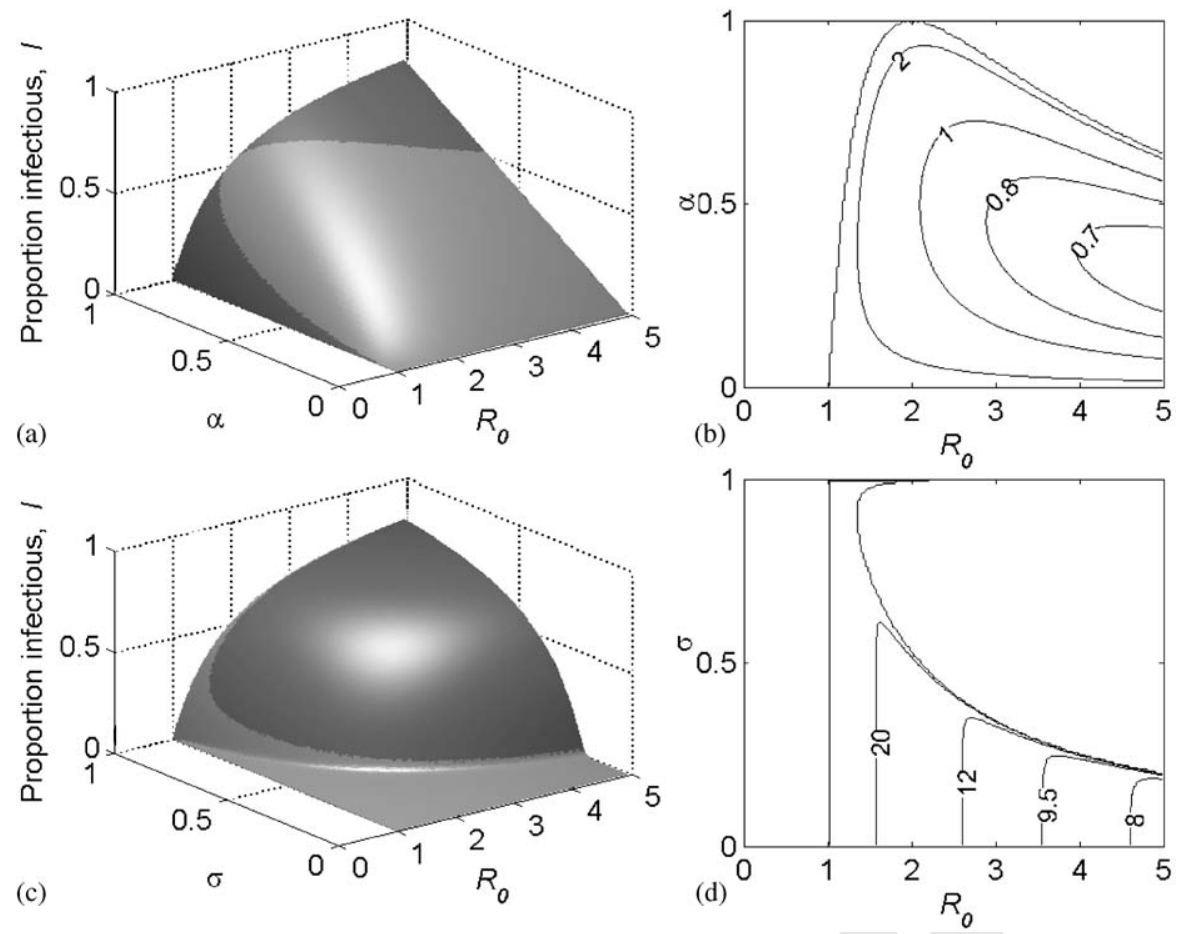

Fig. 4. Equilibria and convergence for the temporary immunity and partial immunity models. (a) and (b) refer to temporary immunity: (a) shows the endemic equilibrium as a function of $R_{0}$ and $\alpha$ indicating whether convergence is by damped oscillations (light grey) or linear decay (dark grey); and (b) shows contour plots for the period of the oscillations in the damped oscillatory region (the labels represent years). (c) and (d) provide the same information for partial immunity: (c) shows the endemic equilibrium as a function of $R_{0}$ and $\sigma$; and (d) shows contour plots for the period of the oscillations.

$\frac{\mathrm{d} S}{\mathrm{~d} t}=e-R_{0} I S-e S+\alpha(1-e)(1-S)$,

$\frac{\mathrm{d} I}{\mathrm{~d} t}=R_{0} I S-I$.

5

The new parameter can take values between 0 and 1 . In the limit $\alpha=0$, the rate of loss of immunity equals the death rate (SIR limit). In the limit $\alpha=1$, the rate of loss of immunity equals the rate of loss of infectiousness (SIS limit). The equilibrium curve for $\alpha=0.04$ is represented as a dashed line in Fig. 3. Fig. 4(a) shows the stable endemic equilibrium as a function of $R_{0}$ and $\alpha$ indicating whether convergence is by damped oscillations (light grey) or linear decay (dark grey). Fig. 4(b) shows the contour plots for the period of the oscillations in the damped oscillatory region (with time rescaled back to years). Naturally, waning of immunity has a crucial impact on the time-scale for potential oscillatory dynamics.

\subsection{Partial immune protection}

There are many infections motivating the study of partial immune protection. Influenza A and B viruses cause the same respiratory disease, but exhibit contrasting evolutionary features (Hay et al., 2001). Partial immunity across antigenic variants is a principal player in models designed to study influenza evolution (Andreasen et al., 1997; Ferguson et al., 2003; Gog and Grenfell, 2002; Gomes et al., 2004b). Other pathogens invoking partial immunity include Neisseria meningitidis (Gupta and Maiden, 2001), Streptococcus pneumoniae (Lipsitch, 1997), and Mycobacterium tuberculosis (Vynnycky and Fine, 1997; Gomes et al., 2004a).

The partial immunity model is represented diagrammatically in Fig. 1(d) and formalized by the system of equations

$$
\begin{aligned}
& \frac{\mathrm{d} S}{\mathrm{~d} t}=e-R_{0} I S-e S, \\
& \frac{\mathrm{d} I}{\mathrm{~d} t}=R_{0} I(S+\sigma(1-S-I))-I .
\end{aligned}
$$

The new assumption is that individuals are protected while infected but regain some susceptibility upon recovery. This susceptibility is reduced by a factor $\sigma$, compared to susceptibility prior to infection. The limit $\sigma=0$ is an SIR model, and the limit $\sigma=1$ is an $S I S$ model. The equilibrium curve for $\sigma=0.4$ is represented as a dotted line in Fig. 3. Fig. 4(c) shows the stable endemic equilibrium as a function of $R_{0}$ and $\sigma$ indicating whether convergence is by damped oscillations (light grey) or linear decay (dark grey). Fig. 4(d) shows the contour plots for the period of the oscillations in the damped oscillatory region (in years). The figure reveals 
1 two clearly distinct types of endemic behaviour: low and potentially oscillatory; and high and steady. Roughly,

3 the first requires that transmissibility is above the threshold for disease persistence $\left(R_{0}>1\right)$, and the second

5 relies on reinfection requiring that transmissibility is above a higher threshold $\left(R_{0}>1 / \sigma\right)$.

\subsection{Temporary-partial immunity}

We used the dynamics of pertussis as an example of temporary immunity, and we evoked a number of infections as examples of partial immunity. However, the transmission dynamics of each of these infections is likely to be associated with a specific combination of both temporary and partial immunity processes, as recently analysed for respiratory syncytial virus (White et al., 2004). We construct a general framework where the contributions of the two mechanisms are combined.

The temporary-partial immunity model is represented by the diagram in Fig. 1(e). As before, $\alpha$ is a parameter that controls the rate of loss of immune protection, and $\sigma$ controls the degree of protection that individuals acquire upon recovery from infection. The model equations are

$\frac{\mathrm{d} S}{\mathrm{~d} t}=e-R_{0} I S-e S+\alpha(1-e)(1-S)$,

$\frac{\mathrm{d} I}{\mathrm{~d} t}=R_{0} I(S+\sigma(1-S-I))-I$.

Fig. 5 illustrates the position of SIR and SIS frameworks in the parameter space $(\alpha, \sigma)$ that characterizes temporary-partial immune protection. The $S I R$ model is retrieved at $\alpha=0$ and $\sigma=0$, and the SIS model corresponds to $\alpha=1$ or $\sigma=1$. The temporary immunity model is retrieved at $\sigma=0$, and the partial immunity model is obtained at $\alpha=0$. As observed in Fig. 4, a temporary immunity mechanism has a strong impact on potential epidemic dynamics, and a partial immunity mechanism induces a second transmission threshold separating regions of low-oscillating and high-steady endemicity. Here, we analyse the combined effects of these two mechanisms. Fig. 6 illustrates how the behaviour of the system depends on $\alpha$ and $\sigma$ for three fixed values of $R_{0}$. Fig. 6(a), (c) and (e) show the stable endemic equilibrium, for $R_{0}=3.5,4.0,4.5$, respectively, indicating whether convergence is by damped oscillations (light grey) or linear decay (dark grey). The respective contour plots for the period of the oscillations are shown in Fig. 6(b), (d) and (f).

\section{Temporary immune protection and vaccination}

We proceed to illustrate the impact of mass vaccination at birth under the temporary immunity model. The model, which assumes that protection induced by the

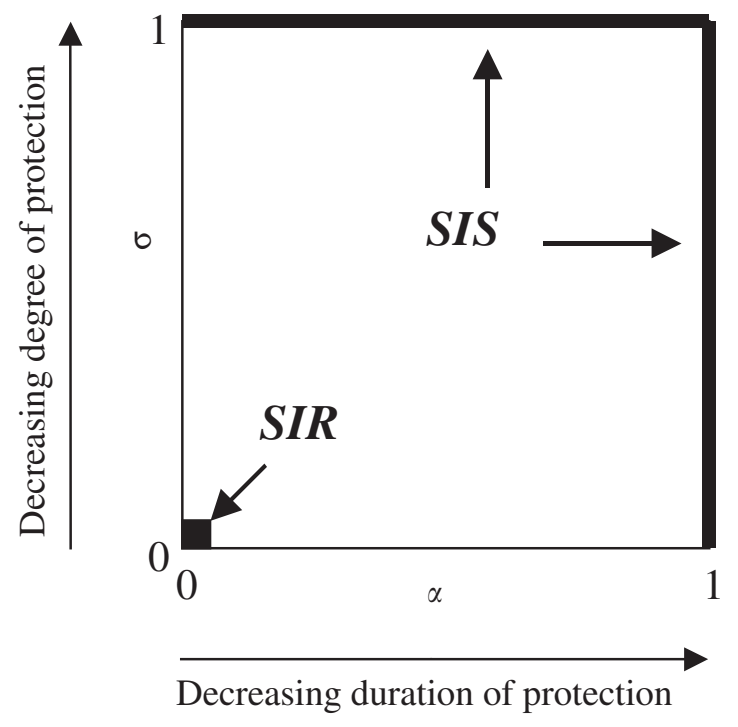

Fig. 5. Schematic diagram illustrating the position of the SIR and SIS frameworks in the parameter space $(\alpha, \sigma)$ characterizing temporarypartial immune protection.

vaccine is equivalent to protection acquired in response to natural infection, is formalized by the system of equations

$$
\begin{aligned}
& \frac{\mathrm{d} S}{\mathrm{~d} t}=(1-v) e-R_{0} I S-e S+\alpha(1-e)(1-S), \\
& \frac{\mathrm{d} I}{\mathrm{~d} t}=R_{0} I S-I,
\end{aligned}
$$

where the new parameter, $v$, represents coverage of the vaccination programme. Fig. 7 illustrates the effect of vaccination in this system when $\alpha=0.0015$. In this case, the average duration of immunity, $1 / \alpha(1-e)$, corresponds to approximately 55 years to represent the loss of immunity commonly observed late in life (which is sometimes observed, even for SIR type infections). The full curves in Fig. 7(a) represent the endemic equilibrium without vaccination (top curve) and when the system is subject to a mass vaccination programme with $90 \%$ coverage (bottom curve). The dashed line represents the unrealistic limit of $100 \%$ vaccination coverage. The dashed line meets the $R_{0}$-axis at the critical value:

$R_{0 \alpha}=1+\frac{e}{\alpha(1-e)}$

implying that eradication is possible only if, in the absence of vaccination, $R_{0}$ is lower than this threshold. For the parameter values set here, $R_{0 \alpha}$ is approximately 1.84. This appears remarkably low when we note that in the idealized situation of lifelong immunity, $\alpha=0$, the eradication threshold would be pushed to infinity, implying that the infection could always be eradicated by vaccination as long as the coverage was sufficiently high (i.e. greater than $1-1 / R_{0}$ ). Fig. 7 (b) shows the result of simulations with two model populations: 


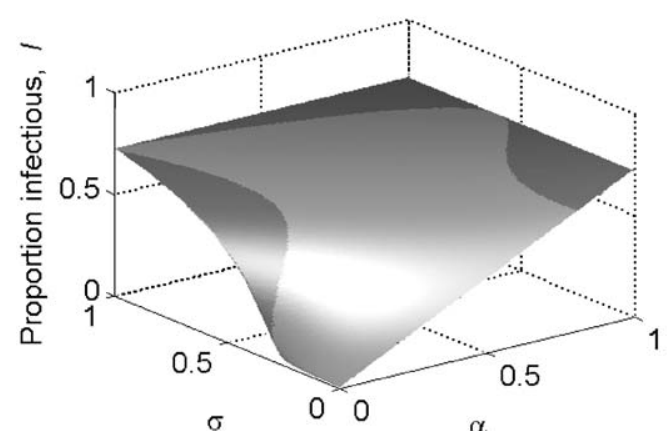

(a)

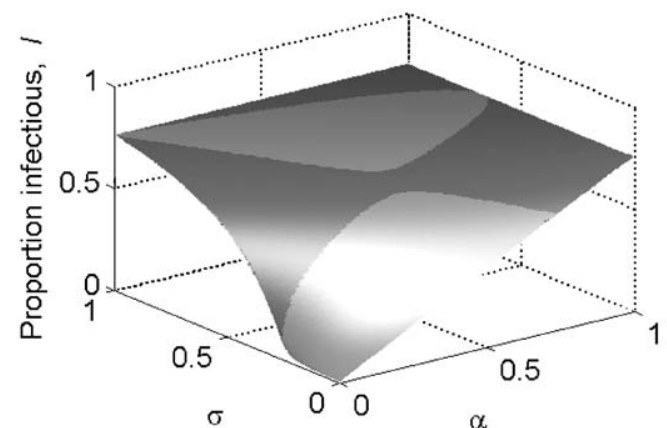

(c)

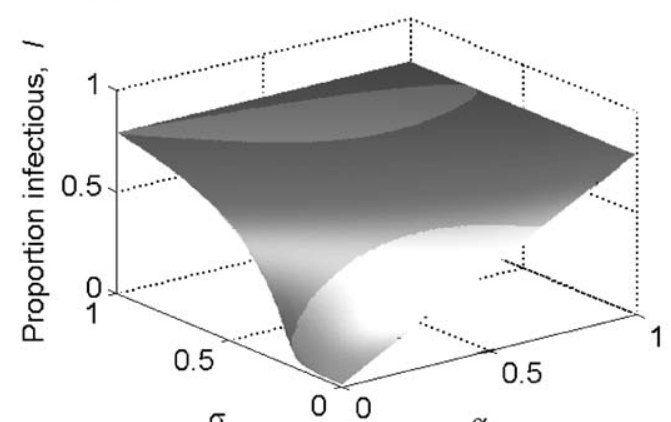

(e)

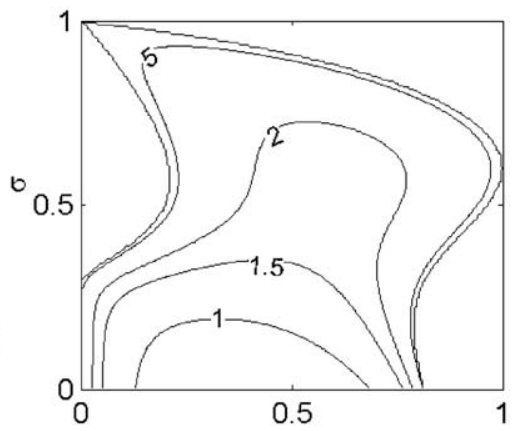

(b)

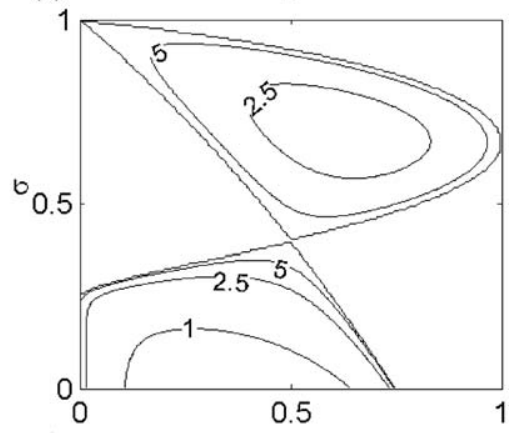

(d)

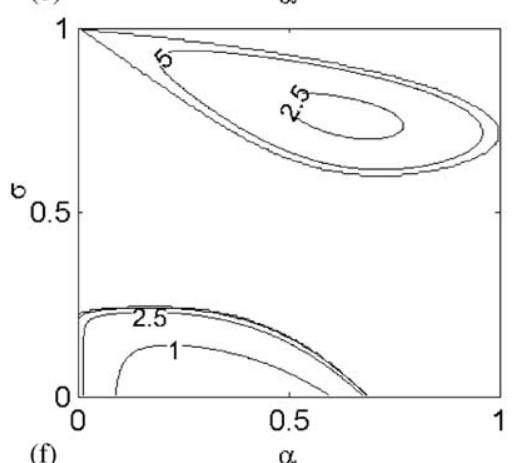
situation of a vaccine that induces as much protection as a previous infection. Then we analyse the impact of more efficacious vaccines.

The main observation from these results is that waning of immunity is a major obstacle to the eradication of infectious diseases. This is illustrated here with a situation where immunity lasts, on average, 55 years and the average lifetime is 70 years. The deterministic models used here predict that, in this scenario, eradication is possible only if $R_{0}$ is below 1.84 . Further investigations with discrete stochastic models may give more optimistic results, but this is beyond the scope of this paper.

\section{Partial immune protection and vaccination}

Here, we illustrate the impact of mass vaccination at birth in the case where both infection and vaccination

\subsection{The reinfection threshold}

Before describing vaccination models, we elaborate on a concept that will be crucial to the analysis - the "reinfection threshold". The full line of Fig. 8 shows how the endemic equilibrium increases with $R_{0}$ when $\sigma=0.25$, revealing a steep increase as transmission crosses

$R_{0 \sigma}=\frac{1}{\sigma}$

which in this case is $R_{0}=4$. This increase is associated with the reinfection threshold. The dotted and dashed 

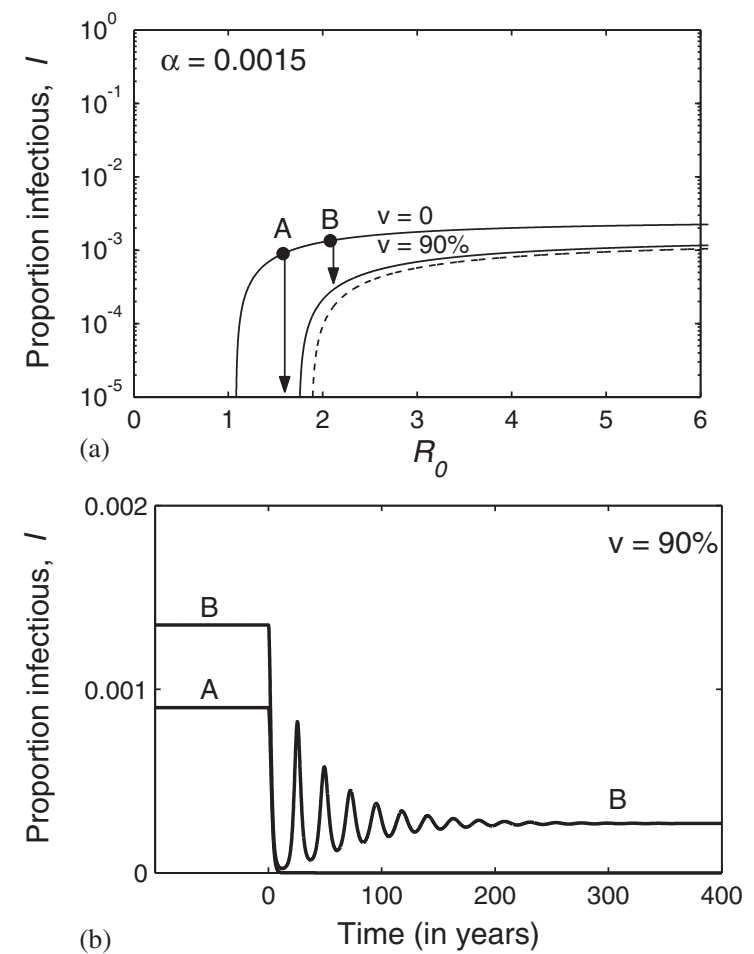

Fig. 7. Temporary protection the global impact of mass vaccination. The rate of loss of immune protection is fixed by setting $\alpha=0.0015$, implying that the average duration of protection of 55 years. (a) shows endemic equilibrium of model (7) as a function of $R_{0}$ : without vaccination (higher full curve), with $90 \%$ vaccination coverage (lower full curve), and with $100 \%$ vaccination coverage (dashed curve). The dots $\mathrm{A}$ and $\mathrm{B}$ represent two model population with different $R_{0}$ : eradication is possible in A, but not in B. (b) simulates the implementation of a vaccination programme (with $90 \%$ coverage) on both populations.

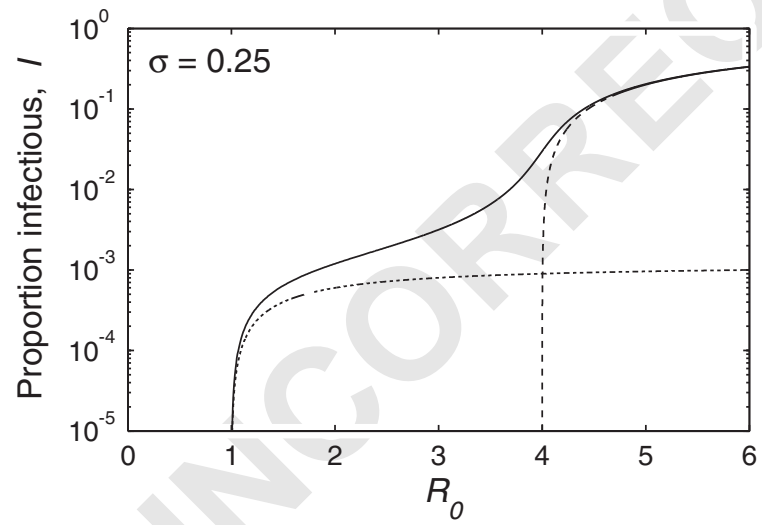

Fig. 8. The reinfection threshold. The full line shows the endemic equilibrium of model (5) as a function of $R_{0}$. The dotted line corresponds to the $S I R$ submodel $\mathrm{d} S / \mathrm{d} t=e-R_{0} I S-e S ; \mathrm{d} I / \mathrm{d} t=R_{0} I S-I$ and represents primary infection. The submodel sets the invasion threshold at $R_{0}=1$. The dashed line corresponds to the $S I S$ submodel $\mathrm{d} I / \mathrm{d} t=\sigma R_{0} I(1-I)-I$ and represents reinfection. By fixing $\sigma=0.25$, the reinfection threshold is set at $R_{0}=1 / \sigma=4$.

lines are plotted to illustrate the contributions of primary infection and reinfection to the overall infection levels, and correspond to the equilibria of two sub- models: the dotted line corresponds to the $S I R$ model $\mathrm{d} S / \mathrm{d} t=e-R_{0} I S-e S ; \mathrm{d} I / \mathrm{d} t=R_{0} I S-I$ and represents primary infection; and the dashed line corresponds to the $S I S$ model $\mathrm{d} I / \mathrm{d} t=\sigma R_{0} I(1-I)-I$ and represents reinfection. The invasion threshold $\left(R_{0}=1\right)$ is associated with the primary infection submodel, and the reinfection threshold $\left(R_{0}=4\right)$ is associated with the reinfection submodel. The full model combines the two dynamical processes, and reveals the potential to convert small baseline variabilities of the transmissibility parameter, $R_{0}$, into large variabilities of infection prevalence.

\subsection{Vaccine inducing protection equivalent to natural infection}

The first vaccination model corresponds to a vaccine that induces an immune response equivalent to that induced by a previous infection. This is formalized by the system of equations

$$
\begin{aligned}
& \frac{\mathrm{d} S}{\mathrm{~d} t}=(1-v) e-R_{0} I S-e S, \\
& \frac{\mathrm{d} I}{\mathrm{~d} t}=R_{0} I(S+\sigma(1-S-I))-I,
\end{aligned}
$$

where $v$ is the vaccination coverage. Fig. 9(a) and (c) illustrates the effect of mass vaccination in this scenario. Note that such a vaccine is protective against primary infection, but not against reinfection. Primary infection is associated with the susceptibility pool $S$; and reinfection associated with the susceptibility pool $S+R$. Vaccination moves individuals from $S$ to $R$ and therefore, essentially reduces the resource for primary infections without directly affecting reinfection. Therefore, it is expected that a mass vaccination programme will be effective below the reinfection threshold, but not above. This is evident from Fig. 9(a), where we show the equilibria without vaccination and with $90 \%$ vaccination coverage (full lines). The dashed line corresponds the unrealistic limit of $100 \%$ vaccination coverage. Three model populations are marked: population A is below the reinfection threshold and infection eradication is expected with the $90 \%$ vaccination programme; population B is also below the reinfection threshold and although $90 \%$ coverage is not sufficient for eradication, a substantial reduction in prevalence is expected; population $\mathrm{C}$ is above the reinfection threshold and the vaccination programme has only a minor effect on the equilibrium prevalence. Fig. 9(c) shows the results of simulating vaccination on the three populations.

The main outcome of this analysis is to demonstrate with a simple model how a vaccination programme can have such variable outcomes. Tuberculosis and the bacille Calmette-Guérin (BCG) vaccine constitute the best documented example of this phenomenon, and this is further discussed in (Gomes et al., 2004a). The variability associated with the reinfection threshold 

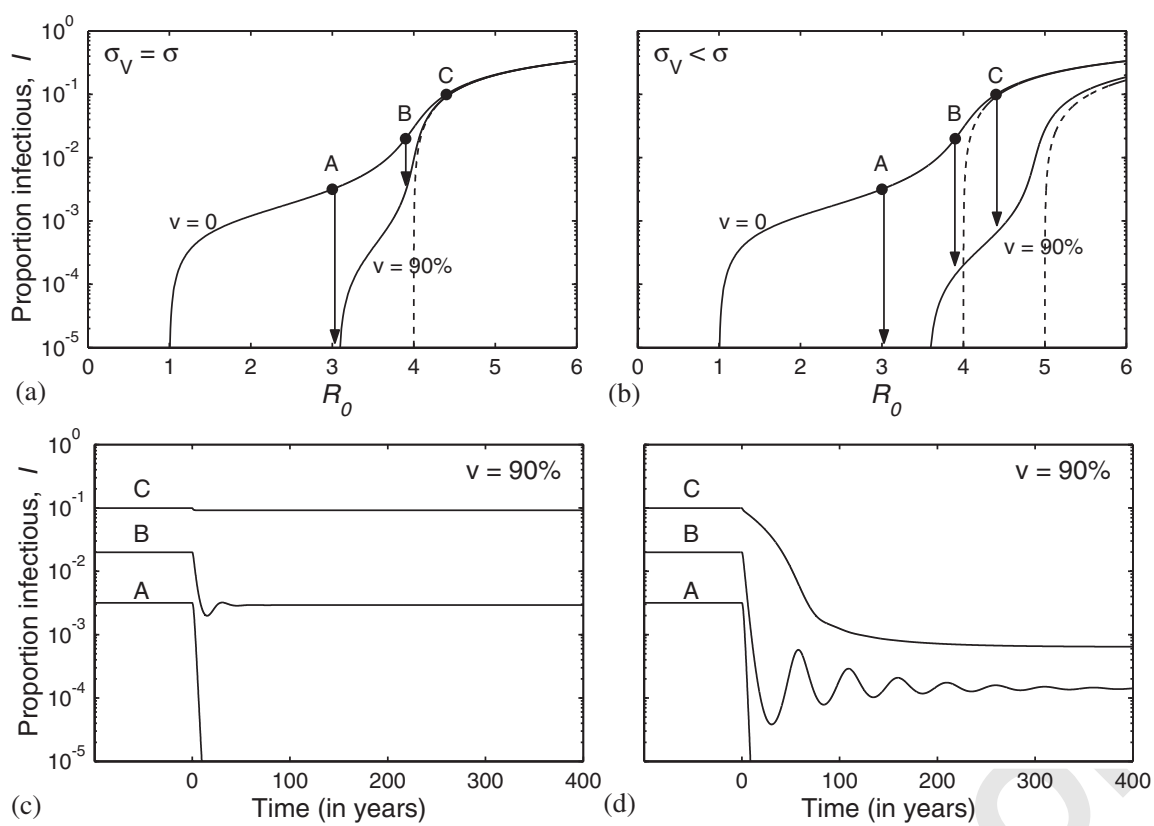

illustrate the impact of two vaccination programmes: (a) and (c) show the result of $90 \%$ coverage with a vaccine that confers as much protection as natural infection (model (8)); (b) and (d) show the result of $90 \%$ coverage with a vaccine that is more potent than natural infection (model (9) with $\left.s_{V}=0.2\right)$. The second vaccine increases the reinfection threshold generating spectacular outcomes in high burden regions that were insensitive to the first vaccine (e.g. population C).

presents serious difficulties to the estimation of vaccine efficacy from field observations, which led to great controversy concerning the use of BCG.

\subsection{Vaccine inducing greater protection natural infection}

A sound understanding of susceptibilities to infection and reinfection is essential to predict the impact of vaccination programmes when immunity is partially protective. The hope is that, where transmissibility is above the reinfection threshold, vaccines that protect more than a natural infection might prove much more effective. This can be demonstrated with the model

$\frac{\mathrm{d} S}{\mathrm{~d} t}=(1-v) e-R_{0} I S-e S$,

$\frac{\mathrm{d} I_{S}}{\mathrm{~d} t}=R_{0} I(S+\sigma R)-I_{S}$

$\frac{\mathrm{d} R}{\mathrm{~d} t}=(1-e) I_{S}-\sigma R_{0} I R-e R$,

$\frac{\mathrm{d} V}{\mathrm{~d} t}=v e+(1-e) I_{V}-\sigma_{V} R_{0} I V-e V$,

$\frac{\mathrm{d} I}{\mathrm{~d} t}=\sigma_{V} R_{0} I V-I_{V}$,

where $\sigma_{V}$ is the factor by which vaccination reduces susceptibility, and here we consider $\sigma_{V}<\sigma$. Fig. 9(b) and (d) illustrates the effect of such vaccine when $\sigma=0.25$ as before and $\sigma_{V}=0.2$. The full lines in Fig. 9(b) represent the pre-vaccination state (as before) and the post- vaccination equilibrium with $90 \%$ coverage. We see that the range of $R_{0}$ where this vaccine has the potential to be highly effective has increased, going up to $1 / \sigma_{V}$, which is $R_{0}=5$ in this case. In other words, if vaccine protection is higher than naturally acquired protection $\left(\sigma_{V}<\sigma\right)$ then vaccination has the power to increase the reinfection threshold to

$R_{0 V}=\frac{1}{\sigma_{V}}$.

From the three model populations used before, it is population $\mathrm{C}$ (where endemicity is highest) who would benefit the most with the development of such vaccine. Fig. 9(d) shows the simulations of the vaccination programme from the time it is introduced until the new equilibrium is reached.

These results have important implications for public health. A great challenge of vaccine development is to supersede the protection provided by natural infection. The reinfection threshold magnifies epidemiological variabilities, but perhaps the most exciting news is that this threshold can be manipulated by vaccines. Vaccines that protect more than naturally acquired infection increase the threshold for reinfection, and may have greater impact than expected in regions afflicted by high burdens of disease. In the case of tuberculosis, a number of strategies for the development of better vaccines are being followed (Britton and Palendira, 2003; Olsen and 
1 Andersen, 2003), and the potential of such interventions cannot be overemphasized (Gomes et al., 2004a).

3

\section{6. Discussion}

7 Since the majority of pathogen infections involve reinfection, the dynamic consequences of any interven-

9 tion on rates of (re)infection should be considered. We suggest that most infections can be classified according

11 to the relative significance of partial and temporary immunity in creating susceptibility to reinfection (Fig.

13 5). Temporary immunity is, to a large extent, determined by the propensity for antigenic change, but also

alteration in host status (e.g. immunosuppression due to other infections). Partial immunity is more dependent on the interaction between static host and pathogen variation: high parasite variation decreasing partial immunity, and high host variation increasing partial immunity. As rates of antigenic change will depend on the variability in the population, one might expect that pathogens occupy the diagonal region in Fig. 5.

The exact mechanism by which individuals regain susceptibility following infection has an important influence on the level of infection maintained in the population and on the potential periodicity of epidemics. Fig. 4(a) and (b) suggests that waning of immunity has a crucial impact on the time-scale for potential inter-epidemic periods. Fig. 4(c) and (d) reveals two clearly distinct types of endemic behaviour: low and high. The first requires that transmissibility is just above $R_{0}=1$. The second relies on reinfection and requires that transmissibility is above $R_{0}=1 / \sigma$. Furthermore, the different mechanisms of immunity failure impact differentially on control strategies.

Many diseases are subject to seasonal forcing of transmission, and this has motivated extensive investigations for the SIR scenario (Keeling et al., 2001). Basically, the resulting inter-epidemic period is inferred from the interplay of the annual cycle and the period of the damped oscillations (Weber et al., 2001). Here we observe that the period for damped oscillations is highly sensitive to the mode of action of immune protection (especially temporary immunity). A combination of these mechanisms with seasonality may be necessary to explain time-scales observed in epidemic cycles.

A more realistic scenario is to consider many levels of susceptibility to infection, or even a continuum. This has been previously implemented (White and Medley, 1998) but analysis has been limited by model complexity. In cases of antigenic diversity, the dynamics of immunity should somewhat correlate with the pathogen evolution, and this has been explored to some extent (Gomes et al., 2002, 2004b; Gog and Grenfell, 2002; Ferguson et al., 2003). In the context of Trypanosoma infections, Coen et al. (2001) attempted to estimate the rate of loss of immunity from seroprevalence data. However, unless primary infection can be distinguished from subsequent infection in some manner (e.g. antibody profile), the parameters for infection rate and immunity loss will be colinear, complicating statistical analysis. A further complication is that the risk of disease on infection might be higher for primary infection (e.g. RSV), or increase with time since last infection or be determined by pathogen (genetic) type. Consequently, the impacts that we show in terms of prevalence and incidence of infection might be different from prevalence and incidence of disease. We have also assumed that subsequent infections are as infectious as primary infections, which is also unlikely to be generally true.

The incidence of pulmonary tuberculosis can vary by two orders of magnitude between different regions of the world, and estimates for the efficacy of the BCG vaccine vary between $0 \%$ and $80 \%$. This scenario is aggravated by an association between high prevalence of infection and low vaccine efficacy (Olsen and Andersen, 2003). It is reasonable to expect vaccination programmes to be less successful in highly endemic regions, and all models discussed here show this effect to some extent. However, the extreme variabilities observed in TB and BCG efficacy are a specificity of the partial immune protection mechanism, due to the reinfection threshold. We have recently proposed this mechanism as an explanation for the BCG discrepancies (Gomes et al., 2004a). Here we show that the conclusions are not specific to tuberculosis but rather, they are a general feature of diseases characterized by partial immune protection and the associated reinfection threshold.

The reinfection threshold represents the transmissibility required to promote recurrent infections. Populations that exceed this threshold, sustain high levels of infection and tend to be insensitive to interventions. Furthermore, the reinfection threshold can be potentially manipulated by vaccination. Vaccines that induce more protection than a natural infection increase the reinfection threshold, providing means for the control of recurrent infection in high-burden regions. Consequently, the partial immune protection framework can serve as a basis to assess the impact of specific vaccines, and to set targets for future vaccine performance in terms of their epidemiological impact (McLean, 1995b). While such vaccines are currently unavailable, efforts can be exerted into manipulating transmission. That is, in populations of high endemicity, improvements in hygiene or reduced crowding could reduce the risk of transmission and therefore the basic reproduction number. If the basic reproduction number is reduced below the reinfection threshold, there would be resulting a drop in prevalence of infection and an increase in apparent vaccination effectiveness. 
Hay, A.J., Gregory, V., Douglas, A.R., Lin, Y.P., 2001. The evolution of human influenza viruses. Philos. Trans. R. Soc. London B 356, $1861-1870$.

We thank Francisco Dionisio and Isabel Gordo for comments on previous versions of this manuscript. This research was initiated while MGMG was a Wellcome Trust Fellow at the University of Warwick, and continued with funding from The Gulbenkian Foundation, and FCT of Portugal. We thank the Wellcome Trust for grant support for LJW.

\section{References}

Anderson, R.M., May, R.M., 1991. Infectious Diseases of Humans: Dynamics and Control. Oxford University Press, Oxford.

Andreasen, V., Lin, J., Levin, S.A., 1997. The dynamics of cocirculating influenza strains confering partial cross-immunity. J. Math. Biol. 35, 825-842.

Britton, W.J., Palendira, U., 2003. Improving vaccines against tuberculosis. Immunol. Cell Biol. 81, 34-45.

Cane, P.A., 2001. Molecular epidemiology of respiratory syncytial virus. Rev. Med. Virol. 11, 103-116.

Coen, P.G., Luckins, A.G., Davison, H.C., Woolhouse, M.E.J., 2001. Trypanosoma evansi in Indonesian buffaloes: evaluation of simple models of natural immunity to infection. Epidemiol. Infect. 126, $111-118$.

Diekmann, O., Heesterbeek, J.A.P., 2000. Mathematical epidemiology of infectious diseases: model building, analysis and interpretation. Mathematical and Computational Biology. Wiley, New York.

Earn, D.J.D., Dushoff, J., Levin, S.A., 2002. Ecology and evolution of the flu. Trends Ecol. Evol. 17, 334-340.

Ferguson, N.M., Galvani, A.P., Bush, R.M., 2003. Ecological and immunological determinants of influenza evolution. Nature 422, $428-433$.

Gog, J.R., Grenfell, B.T., 2002. Dynamics and selection of many-strain pathogens. Proc. Natl Acad. Sci. USA 99, 17209-17214.

Gomes, M.G.M., Medley, G.F., Nokes, D.J., 2002. On the determinants of population structure in antigenically diverse pathogens. Proc. R. Soc. London B 269, 227-233.

Gomes, M.G.M., Franco, A., Gomes, M.C., Medley, G.F., 2004a. The reinfection threshold promotes variability in tuberculosis epidemiology and vaccine efficacy. Proc. R. Soc. London B, in press.

Gomes, M.G.M., Gordo, I., Oliveira-Martins, J.B., 2004b. Reinfection thresholds regulate pathogen diversity: the case of influenza. Submitted for publication.

Grenfell, B.T., Bjornstad, O.N., Kappey, J., 2001. Travelling waves and spatial hierarchies in measles epidemics. Nature 414, 716-723.

Gupta, S., Maiden, M.C.J., 2001. Exploring the evolution of diversity in pathogen populations. Trends Microbiol. 9, 181-185.
Hethcote, H.W., 1999. Simulations of pertussis epidemiology in the United States: effects of adult booster vaccinations. Math. Biosci. $158,47-73$.

Keeling, M.J., Rohani, P., Grenfell, B.T., 2001. Seasonally forced disease dynamics explored as switching between attractors. Physica D 148, 317-335.

Kermack, W.O., McKendrick, A.G., 1927. A contribution to the mathematical theory of epidemics. Proc. R. Soc. A 115, 700-721.

Lipsitch, M., 1997. Vaccination against colonizing bacteria with multiple serotypes. Proc. Natl Acad. Sci. USA 94, 6571-6576.

Liu, W.M., Hethcote, H.W., Levin, S.A., 1987. Dynamical behavior of epidemiological models with nonlinear incidence rates. J. Math. Biol. 25, 359-380.

McLean, A.R., 1995a. After the honeymoon in measles control. Lancet 345, 272.

McLean, A.R., 1995b. Vaccination, evolution and changes in the efficacy of vaccines: a theoretical framework. Proc. R. Soc. London B 261, 389-393.

McLean, A.R., Blower, S.M., 1993. Imperfect vaccines and herd immunity to HIV. Proc. R. Soc. London B 253, 9-13.

Nokes, D.J., Anderson, R.M., 1992. Mathematical models of infectious agent transmission and the impact of mass vaccination. Rev. Med. Microbiol. 3, 187-195.

Nokes, D.J., Anderson, R.M., 1993. Application of mathematical models to the design of immunization strategies. Rev. Med. Microbiol. 4, 187-195.

Olsen, A.W., Andersen, P., 2003. A novel TB vaccine; strategies to combat a complex pathogen. Immunol. Lett. 85, 207-211.

Osborne, K., Gay, N.J., Hesketh, L., Morgan-Capner, P., Miller, E., 2000. Ten years of serological surveillance in England and Wales: methods, results, implications and action. Int. J. Epidemiol. 29, 362-368.

van Boven, M., de Melker, H.E., Schellekens, J.F.P., Kretzschmar, M., 2000. Waning immunity and sub-clinical infection in an epidemic model: implications for pertussis in The Netherlands. Math. Biosci. 164, 161-182.

Vynnycky, E., Fine, P.E.M., 1997. The natural history of tuberculosis: the implications of age-dependent risks of disease and the role of reinfection. Epidemiol. Infect. 119, 183-201.

Weber, A., Weber, M., Milligan, P., 2001. Modelling epidemics caused by respiratory syncytial virus (RSV). Math. Biosci. 172, 95-113.

White, L.J., Medley, G.F., 1998. Microparasite population dynamics and continuous immunity. Proc. R. Soc. London B 265, 19771983.

White, L.J., Mandl, J.N., Gomes, M.G.M., Bodley-Tickell, A.T., Cane, P.A., Perez, P., Siqueira, M.M., Portes, S.A., Straliotto, S.M., Waris, M., Medley, G.F., Nokes, D.J., 2004. Understanding the transmission dynamics of RSV: multiple time series and nested models. Submitted for publication. 